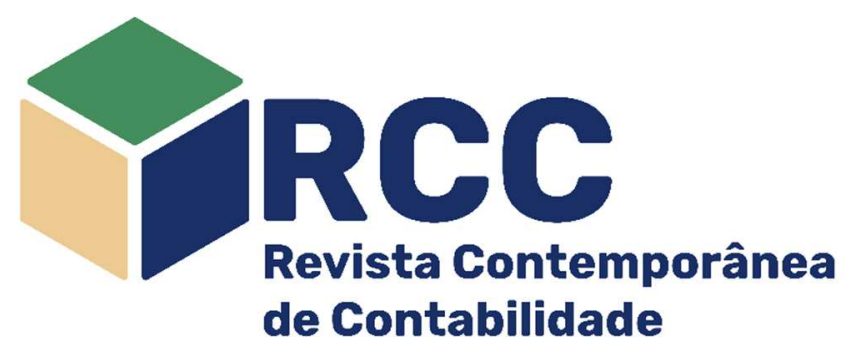

\title{
Conflitos de agência em empresas familiares de capital aberto e a composição do Conselho de Administração
}

\author{
Agency conflicts in publicly traded family businesses and the composition of the Board of Directors

\begin{abstract}
Conflictos de agencia en empresas familiares que cotizan en bolsa y la composición de la Junta Directiva
\end{abstract}

Camila Adam*

Mestranda em Ciências Contábeis (FURB),

Blumenau/SC, Brasil

adam.camila11@gmail.com

https://orcid.org/0000-0001-7404-926X (0)

Daniel Magalhães Mucci

Doutor em Controladoria e Contabilidade (USP) Professor no Departamento de Contabilidade e Atuária

(USP), São Paulo/SP, Brasil danielmmucci@usp.br

https://orcid.org/0000-0002-0658-1470 (1)
Jules Kout Tene Mestrando em Ciências Contábeis (FURB), Blumenau/SC, Brasil tenejules5@yahoo.fr https://orcid.org/0000-0002-0001-9233 (1)

Franciele Beck Doutora em Controladoria e Contabilidade (USP) Professora no Programa de Pós-Graduação em Ciências Contábeis (FURB), Blumenau/SC, Brasil fbeck@furb.br https://orcid.org/0000-0001-7390-5933

Endereço do contato principal para correspondência*
Rua Antônio da Veiga, no 140, Bairro Itoupava Seca, CEP: $89030-903$ - Blumenau/SC, Brasil

\section{Resumo}

O objetivo do estudo é analisar a influência dos conflitos de agência decorrentes da estrutura de propriedade sobre a presença de membros não familiares no Conselho de Administração das empresas familiares brasileiras de capital aberto. A amostra foi composta por 145 empresas familiares de capital aberto. Coletaram-se os dados nos Formulários de Referência divulgados pelas empresas do ano de 2018, utilizandose a regressão linear com o método de Mínimos Quadrados Ordinários (MQO) e logística como procedimento de análise de dados. Os resultados apontam que há uma relação negativa entre o conflito principal-principal e a presença de membros não familiares no Conselho de Administração, bem como a relação positiva entre o conflito family blockholder e presença de membros não familiares no Conselho de Administração. Ademais, esse estudo traz como implicação evidências de que a composição do Conselho de Administração é influenciada por conflitos de agência relacionados à estrutura de propriedade das empresas familiares.

Palavras-chave: Conflitos de agência; Empresas familiares; Composição Conselho de Administração

\section{Abstract}

The aim of the study is to analyze the influence of agency conflicts arising from ownership structure on the presence of non-family members on the Board of Directors of Brazilian family companies. The sample consisted of 145 publicly traded family businesses. The data were collected in the Reference Forms released by the companies of the year 2018. Linear regression with the Ordinary Least Squares (OLS) method and logistics was used as a data analysis procedure. The results indicate that there is a negative relationship between the principal-principal conflict and the presence of non-family members on the Board of Directors and a positive relationship between the family blockholder conflict and the presence of non-family members on the Board of Directors. This study implies evidence that the composition of the Board of Directors is influenced by agency conflicts related to the ownership structure of family businesses.

Keywords: Agency conflicts; Family businesses; Composition of the Board of Directors

\section{Resumen}

El objetivo del estudio es analizar la influencia de los conflictos de agencia que surgen de la estructura de propiedad sobre la presencia de miembros desconocidos en el Consejo de Administración de empresas 
familiares brasileñas que cotizan en bolsa. La muestra consistió en 145 empresas familiares que cotizan en bolsa. Los datos se recopilaron en los Formularios de Referencia publicados por las empresas en el año 2018. La regresión lineal utilizando el método de Mínimos Cuadrados Ordinarios (MCO) y la logística se utilizó como un procedimiento de análisis de datos. Los resultados indican que existe una relación negativa entre el conflicto principal-principal y la presencia de miembros no familiares en la Junta de Directores y una relación positiva entre el conflicto family blockholder y la presencia de miembros no familiares en la Junta de Directores. Este estudio implica evidencia de que la composición de la Junta Directiva está influenciada por conflictos de agencia relacionados con la estructura de propiedad de las empresas familiares.

Palabras clave: Conflictos de agencia; Empresas familiares; Composición de la Junta Directiva

\section{Introdução}

A Teoria da Agência postula sobre o compartilhamento de riscos entre indivíduos, tratando especificamente sobre o problema de agência que surge no relacionamento entre principal e agente (JENSEN; MECKLING, 1976). Conforme Eisenhardt (1989), a Teoria da Agência objetiva resolver dois problemas que decorrem dos referidos relacionamentos; o primeiro refere-se aos objetivos conflitantes entre o principal e o agente, sendo complexa e onerosa a fiscalização das atividades do agente pelo principal; o segundo aborda acerca dos comportamentos discordantes entre principal e agente quanto à preferência de risco, levando a diferentes preferências de ações. Dentro dessa relação, entre o principal e o agente, ressaltase também a ocorrência da assimetria de informação, em que o agente tem acesso a informações que 0 principal não tem e vice-versa, pois o primeiro está presente no dia a dia do negócio.

Conforme Chrisman, Chua e Litz (2004), parte da literatura pressupõe que quando não houver separação entre a propriedade e a administração, os problemas de agência não existem ou são reduzidos. Nesse contexto, enquadram-se as empresas familiares em que se presume a unidade entre os interesses dos proprietários-gerentes (CARNEY, 2005). Consoante Shyu (2011), o controle familiar é significativo na redução dos problemas de agência Tipo I (principal e agente), uma vez que os membros da família têm incentivos para fiscalizar os gerentes, ao passo que as riquezas da família estão relacionadas ao desempenho da empresa.

No entanto, estudos também consideram que, na maioria dos casos, as partes relacionadas terão interesses diferentes, acarretando problemas de agência específicos de empresa familiar (CHRISMAN; CHUA; LITZ, 2004). Nesse sentido, destaca-se que as famílias são compostas por diferentes integrantes, que possuem objetivos pessoais próprios e perspectivas diferentes quanto ao negócio da família (BERTRAND et al., 2008). Dentre os conflitos, evidencia-se o conflito entre acionistas majoritários e minoritários (conflito principal-principal) e o conflito derivado da participação de mais de uma família sem laços consanguíneos na propriedade e no controle da empresa (conflito family blockholder) (SCHULZE; LUBATKIN; DINO, 2003; ZELLWEGER; KAMMERLANDER, 2015).

O conflito de agência de tipo principal-principal acontece entre acionistas majoritários e minoritários e pressupõe que os majoritários expropriam recursos dos minoritários. Já, o conflito family blockholder surge quando houver divergência de interesses entre as famílias proprietárias ou aquelas que se uniram para ter o controle da empresa (ZELLWEGER; KAMMERLANDER, 2015), em se tratando de grupos de controle que exercem influência significativa sobre o negócio.

Para alinhar os interesses do principal com as ações tomadas pelos agentes, empregam-se nas empresas mecanismos de governança corporativa. Haja vista que, de acordo com o Instituto Brasileiro de Governança Corporativa - IBGC (2019), a governança corporativa nas empresas familiares, além de tratar do relacionamento entre propriedade e gestão, aborda questões referentes aos objetivos, aos valores e aos princípios que guiam a empresa, bem como os mecanismos de governança mais usuais, por exemplo, os Conselhos de Administração e diretorias, as estruturas de governança familiar contemplam, ainda, reunião e/ou assembleia familiar, conselho de família, family office e comitê de sócios.

Segundo Costa, Sampaio e Flores (2019), Briano-Turrent e Poletti-Hughes (2017), o Conselho de Administração é um dos mecanismos mais importantes de governança corporativa nas empresas, uma vez que é o foco principal deste trabalho. Nas empresas familiares, o Conselho de Administração alinha os interesses de membros familiares e não familiares com a finalidade de evitar a sobreposição dos interesses familiares aos dos acionistas externos. Assim, o mecanismo utilizado é a presença de membros independentes no Conselho de Administração, os quais medeiam os conflitos entre os membros da família e destes com aqueles (LAFFRANCHINI; BRAUN, 2014).

Conforme apresentado, a fim de mitigar os conflitos de interesses entre os proprietários majoritários e minoritários e entre family blockholders, pode-se ter o ingresso de membros não familiares nos Conselhos de Administração das empresas. Frente a esse contexto, o estudo apresenta a seguinte questão de pesquisa: qual a influência dos conflitos de agência decorrentes da estrutura de propriedade sobre a presença de membros não familiares no Conselho de Administração das empresas familiares brasileiras de capital aberto? Dessa forma, o objetivo é analisar a influência dos conflitos de agência decorrentes da estrutura de 
propriedade sobre a presença de membros não familiares no Conselho de Administração das empresas familiares brasileiras de capital aberto.

Embora a presença de membros não-familiares no Conselho de Administração tenha sido considerada importante para a sobrevivência e para o crescimento das empresas familiares (FANG et al., 2016; HIEBL; LI, 2018), poucas pesquisas analisam como os problemas de agência estão associados à presença de executivos não-familiares. Assim, pesquisas nesse sentido são interessantes para identificar qual a relação entre os conflitos de agência decorrentes da estrutura acionária e a composição do Conselho de Administração nas empresas familiares.

Ademais, estudos sobre governança em empresas familiares no Brasil, também, são escassos, sendo importante investigar as particularidades da estrutura de propriedade delas (BRESSAN et al., 2019). Dentre os estudos nacionais que versam sobre a temática, salienta-se os que investigaram os aspectos positivos dos conflitos em Conselhos de Administração (KARAM; MACHADO FILHO; ABIB, 2019), a participação feminina no Conselho de Administração (COSTA; SAMPAIO; FLORES, 2019), os mecanismos externos de governança e a alavancagem corporativa (MENDONÇA; MARTINS; TERRA, 2019) e a aplicação do Acordo de Quotistas como mecanismo interno de governança (KLEINERT; LANA; FLORIANI, 2019).

Ressalta-se, ainda, que a temática estudada junto aos elementos que são trabalhados, podem ser fundamentais ao desempenho das empresas familiares, o que justifica a importância do estudo também para o ambiente organizacional. Além disso, levando-se em consideração, conforme a literatura, pesquisas que estudam a governança corporativa como mecanismo para mitigar os conflitos de agência nas empresas familiares são muito relevantes e geram contribuições tanto para o ambiente acadêmico como para as organizações.

O estudo contribui para a literatura sobre empresas familiares e problemas de agência- governança corporativa - de várias maneiras. Em primeiro lugar, o estudo trata de problemas de agência específicos das empresas familiares, permitindo uma melhor compreensão dos tipos de conflitos principal-principal e family blockholders que ocorrem nas empresas familiares de capital aberto no Brasil. Em segundo lugar, identificamse as variáveis que determinam a presença de executivos não familiares no Conselho de Administração das empresas familiares e verifica se essas estão alinhadas com os problemas de agência enfrentados pelas empresas.

\section{Revisão da Literatura e Hipóteses de Pesquisa}

\subsection{Problemas de agência na dimensão da propriedade}

A Teoria da Agência, conforme apresentada por Jensen e Meckling (1976), trata do conflito tradicional de agência ou conflito principal-agente que ocorre entre acionistas e administradores, com foco na minimização dos problemas de comportamento oportunista dos agentes. Os conflitos de agência resultam em custos de agência que geralmente surgem devido ao interesse próprio e à tomada de decisões dos indivíduos orientados para as próprias preferências (EISENHARDT, 1989). Em virtude disso, os custos ocorrem devido a diferentes preferências e assimetrias de informação entre o proprietário (principal) e a administração (agente) (JENSEN; MECKLING, 1976).

Para as empresas familiares, muitas vezes se assume que a propriedade e a gestão estão alinhadas dentro da mesma família ou mesmo atribuídas a uma mesma pessoa (CHUA; CHRISMAN; SHARMA, 1999). Segundo Jensen e Meckling (1976), esse alinhamento levaria a evitar custos de agência; no entanto, a influência de questões que envolvam a família nestas empresas cria uma estrutura mais complexa de preferências individuais (CHRISMAN; CHUA; LITZ, 2004). Schulze, Lubatkin e Dino (2003) apontaram, por exemplo, os aspectos relacionais e altruístas como fatores que desempenham um papel importante na tomada de decisões e, assim, geram outras fontes de custos de agência.

De acordo com Zellweger e Kammerlander (2015), as empresas familiares são sujeitas a diversos tipos de conflitos de agência. Além do conflito tradicional de agência ou de conflito de agência de tipo I entre principal e agente, descrito por Jensen e Meckling (1976), pode-se encontrar nas empresas familiares conflitos do tipo II, entre principal-principal, ou seja, entre acionistas majoritários e minoritários (ZELLWEGER; KAMMERLANDER, 2015) e conflitos devido a acordos entre acionistas familiares também tratado na literatura como family blockholder (ZELLWEGER; KAMMERLANDER, 2015), os quais são discutidos neste estudo.

À medida que as empresas familiares crescem, há uma indicação para a profissionalização da estrutura administrativa do negócio, com o intuito de se reduzir as possiblidades de influência tendenciosa dos membros familiares na tomada de decisões da empresa, em virtude da posição de controle dos ativos que esses ocupam (CARNEY, 2005). Como descrito por Chrisman, Chua e Litz (2004), tanto os proprietários familiares entre si quanto os agentes podem exibir preferências distintas em relação a vários assuntos, surgindo os conflitos. Nesse ponto, surge a decisão do emprego de membros não familiares na empresa, com o objetivo de minimizar os conflitos de agência. 


\subsection{Determinantes do Conselho de Administração}

Hiebl e Li (2018) realizaram uma revisão de literatura dos principais estudos que discutiram sobre a composição do Conselho de Administração, com enfoque em empresas familiares cuja gestão está parcial ou totalmente nas mãos de gestores não-familiares - non-family members (NFMs). Tais autores distinguiram três motivos do envolvimento do NFMs em empresas familiares: antecedentes dos proprietários da família, antecedentes do negócio e antecedentes dos proprietários não familiares. Em relação aos antecedentes da família, a contratação de NFMs pode ser influenciada pela necessidade de profissional com conhecimento e experiência (LIN; HU, 2007; ZONA, 2016) e para defender os interesses familiares (BLUMENTRITT; KEYT; ASTRACHAN, 2007; HIEBL, 2014).

No que diz respeito aos antecedentes do negócio, a competitividade do mercado (LIN; HU, 2007), o tamanho da empresa (FANG et al., 2016) bem como a alta capacidade financeira (BENNEDSEN et al., 2007) aumentam a probabilidade de emprego de NFMs nas empresas familiares. Por fim, em relação aos antecedentes dos proprietários não familiares, a presença de proprietários não familiares em empresas familiares aumenta a possibilidade de emprego de NFMs (BERENBEIM, 1990) para defender os interesses principalmente quando a propriedade da empresa é dominada por membros da família (HILLIER; MCCOLGAN, 2009).

Da mesma forma, o estudo de Tabor et al. (2018) fez uma revisão de literatura de 82 artigos sobre membros não pertencentes a famílias que foram publicados em 34 periódicos nas últimas três décadas. Os autores sintetizaram a literatura sobre contratação de NFMs nas empresas familiares em três temas sobrepostos. O primeiro trata das considerações de pré-emprego (formalização, cultura, compensação e antecedentes/experiência e busca por um profissional externo à família); a segunda refere-se às considerações de emprego (justiça e socialização), e a terceira, dos resultados do emprego de NFMs (desempenho da empresa e metas centradas na família).

No âmbito nacional, a literatura sobre governança corporativa e Conselho de Administração versa sobre diferentes aspectos. O estudo de Karam, Machado Filho e Abib (2019) elaborou um arcabouço teórico que busca destacar os aspectos positivos dos conflitos em Conselhos de Administração de empresas familiares. Por outro lado, Costa, Sampaio e Flores (2019) investigaram se a participação feminina no Conselho de Administração de Empresas tem relação com o desempenho financeiro e risco. Mendonça, Martins e Terra (2019) investigaram em que medida os mecanismos de governança externos à firma e relacionados à proteção dos acionistas e aos direitos dos credores afetam a alavancagem corporativa. Enquanto Kleinert, Lana e Floriani (2019) apresentaram o desenvolvimento e a aplicação do Acordo de Quotistas como mecanismo interno de governança corporativa para mitigar conflitos em uma pequena empresa do setor de desenvolvimento de softwares.

Desse modo, percebe-se o interesse da área por mais compreensão das características de governança corporativa em empresas familiares. O presente estudo, em específico, busca avançar essa discussão focalizando a influência dos conflitos de agência decorrentes da estrutura de propriedade sobre a presença de membros não familiares no Conselho de Administração das empresas familiares brasileiras de capital aberto. Na próxima subseção, serão desenvolvidas as hipóteses da pesquisa.

\subsection{Hipóteses de pesquisa}

A concentração de propriedade nas empresas familiares proporciona poder e influência à família para tomar decisões sobre questões estratégicas, composição dos conselhos e mecanismos de governança (VILLALONGA; AMIT, 2006). Nesse ambiente, é propício surgir o conflito de agência principal-principal ou problema de agência do Tipo II, que resulta das divergências de interesses entre os acionistas majoritários e minoritários. Diante da concentração de propriedade e controle, a família pode utilizar da fraca proteção legal dos acionistas minoritários para se apropriar de benefícios privados (YOUNG et al., 2008).

De um lado, o controle familiar pode reduzir os custos de agência, ajudando a alinhar a propriedade ao controle (JENSEN; MECKLING, 1976) e, por outro lado, o controle familiar pode aumentar a probabilidade de expropriação de recursos de acionistas minoritários não-familiares e prejudicar o desempenho, uma vez que os proprietários da família podem nomear membros da família não qualificados para postos-chave (ZELLWEGER; KAMMERLANDER, 2015). Os conflitos entre principal e principal alteram a dinâmica do processo de governança corporativa que, por sua vez, exigem tratamentos diferentes daqueles que lidam com conflitos entre o principal e o agente (YOUNG et al., 2008).

Uma forma de reduzir essas pressões entre os acionistas majoritários e minoritários é a presença de membros não familiares no Conselho de Administração (ILHAN-NAS et al., 2018), visto que a presença de membros não familiares desempenha um papel de proteção dos interesses dos acionistas minoritários em relação à família proprietária (MORCK; YEUNG, 2003; PENG, 2004; RHOADES; RECHNER, 2001).

No entanto, para não reduzirem a influência e o controle sobre os assuntos das empresas, os proprietários da família tendem a adotar mecanismos de governança que fortalecem seu poder, o que não ocorre com o ingresso de membros não familiares no Conselho de Administração (KABBACH DE CASTRO; AGUILERA; CRESPÍ-CLADERA, 2017). Assim, as famílias proprietárias encaram o Conselho de 
Administração como um meio de garantir o controle sobre a empresa (SELEKLER-GOKSEN; YILDIRIMOKTEM, 2009).

Quando a família possui mais participações na empresa, os membros dela possuem a expectativa de controlar os processos de tomada de decisão de modo a preservar a influência. Ademais, o desejo de controle da família é impulsionado pelo apego emocional da família à empresa e é vital mantê-la sob propriedade da família para as gerações futuras (KABBACH DE CASTRO; AGUILERA; CRESPÍ-CLADERA, 2017).

Diante disso, entende-se que uma das estratégias para reduzir o conflito principal-principal em uma empresa familiar de capital aberto (com a presença de acionistas minoritários) seja incorporar membros não familiares no Conselho de Administração. Contudo, a presença de membros não familiares acaba abrindo espaço para que os interesses dos acionistas minoritários sejam resguardados nas tomadas de decisão e, como há o intuito de manter o controle e a influência familiar na empresa, o ingresso de membros não familiares tende a ser contido pela família. Nesse ensejo, apresenta-se a primeira hipótese desta pesquisa:

$H_{1}$ : Há uma associação negativa entre o conflito principal-principal e a presença de membros não familiares no Conselho de Administração de empresas familiares de capital aberto.

Algumas empresas familiares possuem a participação de mais de uma família na propriedade da empresa. Nesse tipo de empresa familiar, a propriedade e o controle são divididos entre duas ou mais famílias que não são relacionadas (PIEPER et al., 2015). Nesse sentido, cada bloco de controle familiar, controla um número substancial de ações da empresa e, portanto, tende a influenciar decisões estratégicas importantes e reforçar suas preferências individuais. Nesse contexto, podem ocorrer os conflitos de agência de tipo family blockholder que remetem à presença de várias famílias proprietárias com objetivos próprios no controle da empresa (ZELLWEGER; KAMMERLANDER, 2015).

Conforme Duran e Ortiz (2020), a participação de mais famílias na propriedade de uma empresa leva a maior diversidade de perspectivas e interesses, que podem gerar conflitos se não forem continuamente negociados. Nesse ambiente, cada bloco familiar tem o incentivo de monitorar as atividades das outras famílias controladoras a fim de garantir que seus interesses não sejam prejudicados, tendo em vista que esses conflitos lesam os benefícios vinculados ao exercício conjunto do poder como um grupo unificado de acionistas e podem ser particularmente caros para a própria família. Além disso, os confrontos entre os defensores da família aumentam ainda mais as dificuldades e os custos, criando conflitos de lealdade entre os diretores sobre quais visões dos proprietários deve seguir, bem como uma atmosfera de desconfiança e incerteza sobre o futuro da empresa (ZELLWEGER; KAMMERLANDER, 2015).

Uma forma frequentemente observada para as famílias restringirem os conflitos family blockholder e garantir a proteção dos seus interesses particulares é a contratação de NFMs com o intuito de ocupar os conselhos na empresa (HIEBL; LI, 2018). A presença de NFMs pode aliviar os conflitos family blockholder, pois ajuda no alinhamento dos interesses divergentes entre as famílias proprietárias e cria um amortecedor entre os proprietários dela e seus ativos, a fim de evitar a interferência descoordenada das famílias proprietárias no negócio (ZELLWEGER; KAMMERLANDER, 2015). Assim, diante do conflito family blockholder, há o incentivo para o ingresso de membros não familiares no Conselho de Administração, com o propósito de mitigar o conflito de interesses entre cada bloco familiar. Sendo assim, a segunda hipótese de pesquisa é formada:

$\mathrm{H}_{2}$ : Há uma associação positiva entre o conflito family blockholder e a presença de membros não familiares no Conselho de Administração de empresas familiares de capital aberto.

\section{Procedimentos Metodológicos}

A pesquisa caracteriza-se quanto ao problema como quantitativa, em relação aos procedimentos documental e no tocante aos objetivos como descritiva.

\subsection{População e amostra}

A população do estudo compreende as empresas familiares brasileiras de capital aberto com ações negociadas na bolsa de valores B3. Já a amostra do estudo foi formada por 145 empresas, após a exclusão das empresas multinacionais de capital estrangeiro e de estatais, visto não se enquadrarem nos parâmetros do estudo. A pesquisa considerou como definição operacional da empresa familiar aquela em que os membros da família detêm $20 \%$ ou mais das ações ordinárias da empresa, segundo a lógica da propriedade familiar. Essa definição operacional é comumente usada para empresas de capital aberto (SINGLA; VELIYATH; GEORGE, 2014; PURKAYASTHA; VELIYATH; GEORGE, 2019; VAZQUEZ; CARRERA; CORNEJO, 2020).

Os dados foram coletados nos Formulários de Referência divulgados pelas empresas familiares da amostra para o ano de 2018, disponíveis no site da bolsa de valores B3 e a coleta ocorreu durante o período de 25 de março de 2019 a 17 de julho de 2019. 
Para garantir a qualidade dos dados, durante o processo de coleta de dados, os pesquisadores realizaram o procedimento de coleta e de conferência simultânea, em que após a coleta e a tabulação realizada por um dos pesquisadores, ocorreu a conferência destes dados por outro pesquisador, visto 0 processo ter sido operacional.

\subsection{Instrumentos e variáveis da pesquisa}

Quanto às variáveis do estudo, destaca-se que para a mensuração da variável dependente, empregou-se a proxy presença de membros não familiares no Conselho de Administração. Codificando 1 se o presidente do Conselho de Administração da empresa era membro não familiar e 0 caso contrário. Em seguida, verificou-se a proporção de membros não familiares em relação ao número total de membros no Conselho de Administração da empresa. Para a definição de membro não familiar, analisou-se o sobrenome dos membros do Conselho de Administração (por exemplo, GARCIA-CASTRO; CASASOLA, 2011; BRIANOTURRENT; POLETTI-HUGHES, 2017; DURAN; ORTIZ, 2020) e as relações familiares desses membros, respectivamente nos itens 12.5/6 e 12.9 do Formulário de Referência.

No tocante às variáveis independentes, combinaram-se duas variáveis para construir a proxy do conflito principal-principal, essa combinação foi feita a partir da análise de componentes principais de duas variáveis relacionadas à estrutura acionária das empresas, percentual de ações ON do maior acionista e percentual de ações ON do segundo maior acionista, proxy adaptada dos estudos de Renders e Gaeremynck (2012) e Purkayastha, Veliyath e George (2019). O conflito family blockholder foi mensurado a partir de uma proxy dummy, codificando 1 se a propriedade da empresa estava sob controle de mais de uma família sem laços consanguíneos e 0 caso estivesse sob influência ou controle de apenas um núcleo familiar. Para a análise da existência de mais de uma família controladora, analisaram-se o sobrenome dos acionistas (GARCIA-CASTRO; CASASOLA, 2011; BRIANO-TURRENT; POLETTI-HUGHES, 2017; DURAN; ORTIZ, 2020) e o percentual de ações ordinárias no item 15.1/2 e as relações familiares no item 12.9 do Formulário de Referência.

Como variáveis de controle, o estudo utilizou as seguintes informações de cada companhia: idade e tamanho da empresa a partir do logaritmo da receita de vendas, conforme os estudos de Purkayastha, Veliyath e George (2019) e Vazquez, Carrera e Cornejo (2020), e as variáveis relacionadas ao nível de governança, nível 1, nível 2 e novo mercado, assim como a existência de acordo de acionistas. Vale destacar que a categoria base para as variáveis relacionadas ao nível de governança foi o nível tradicional. A Tabela 1 apresenta a descrição das variáveis.

Tabela 1 - Descrição das variáveis

\begin{tabular}{|c|c|c|c|}
\hline Variável & \begin{tabular}{|c|} 
Descrição \\
\end{tabular} & Métrica & Referências \\
\hline \multicolumn{4}{|c|}{ Dependente - Composição do Conselho de Administração } \\
\hline PCANF & $\begin{array}{|lrrr|}\text { Proporção de } & \text { membros } & \text { não } \\
\text { familiares no } & \text { Conselho } & \text { de } \\
\text { Administração } & & \\
\end{array}$ & $\begin{array}{l}\text { Razão entre a quantidade de membros } \\
\text { não familiares e o total de membros no } \\
\text { Conselho de Administração. }\end{array}$ & \multirow{2}{*}{$\begin{array}{l}\text { Garcia-Castro e Casasola } \\
\text { (2011) }\end{array}$} \\
\hline PRCANF & $\begin{array}{l}\text { Presidente do Conselho de } \\
\text { Administração não é da família }\end{array}$ & $\begin{array}{l}\text { Dummy: } 1 \text { se o Presidente do Conselho } \\
\text { de Administração não for da família e } 0 \\
\text { caso contrário. }\end{array}$ & \\
\hline \multicolumn{4}{|c|}{ Independente - Conflitos de Agência } \\
\hline CONFLITO_PP & Conflito principal-principal & $\begin{array}{c}\text { Combinação de duas variáveis: a) } \\
\text { percentual de ações ON* do maior } \\
\text { acionista e b) percentual de ações ON } \\
\text { do segundo maior acionista. }\end{array}$ & $\begin{array}{c}\text { Adaptado de Renders e } \\
\text { Gaeremynck (2012); } \\
\text { Purkayastha, Veliyath e George } \\
(2019)^{\text {a }} \\
\end{array}$ \\
\hline CONFLITO_FB & Conflito family blockholder & $\begin{array}{c}\text { Dummy: } 1 \text { se houver mais de uma } \\
\text { família controladora na empresa e zero } \\
\text { caso contrário. }\end{array}$ & $\begin{array}{l}\text { Pieper et al. (2015); Duran e } \\
\text { Ortiz (2020) }\end{array}$ \\
\hline \multicolumn{4}{|c|}{ (2) } \\
\hline IE & Idade da empresa & $\begin{array}{l}\text { Anos de atividade da empresa desde } \\
\text { sua constituição. }\end{array}$ & $\begin{array}{l}\text { Purkayastha, Veliyath e George } \\
\text { (2019); Vazquez, Carrera e }\end{array}$ \\
\hline TAM & Tamanho & Logaritmo da receita de vendas. & Cornejo (2020) \\
\hline N1 & Nível 1 de governança & $\begin{array}{c}\text { Dummy: } 1 \text { se a empresa estiver no } \\
\text { Nível } 1 \text { de governança e } 0 \text { caso } \\
\text { contrário. }\end{array}$ & \multirow{4}{*}{$\begin{array}{l}\text { Regulamentos de listagem dos } \\
\text { níveis de governança da B3. }\end{array}$} \\
\hline N2 & Nível 2 de governança & $\begin{array}{c}\text { Dummy: } 1 \text { se a empresa estiver no } \\
\text { Nível } 2 \text { de governança e } 0 \text { caso } \\
\text { contrário. }\end{array}$ & \\
\hline NM & Novo mercado & $\begin{array}{l}\text { Dummy: } 1 \text { se a empresa estiver no } \\
\text { Novo Mercado e } 0 \text { caso contrário. }\end{array}$ & \\
\hline AA & Acordo de acionistas & $\begin{array}{l}\text { Dummy: } 1 \text { se a empresa possui acordo } \\
\text { de acionistas, e } 0 \text { caso contrário. }\end{array}$ & \\
\hline
\end{tabular}

Nota. *O estudo teve foco nas ações ON por concederem direito ao voto e à indicação de membros para o Conselho de Administração. a Os estudos captaram duas variáveis adicionais na análise de componentes principais, uma variável dummy indicando se a empresa possui dual class shares e outra variável dummy, se os direitos do maior acionista excedem os direitos de fluxo de caixa em mais de $10 \%$. Como o objetivo do estudo está voltado à concentração acionária dos acionistas majoritários como principal indicador do conflito principal-principal, utilizou-se apenas as variáveis indicadas na Tabela 1.

Fonte: Elaborado pelos autores. 
Para a análise dos dados, empregaram-se dois tipos de regressão no software STATA ${ }^{\circledR}$, a regressão linear com o método de Mínimos Quadrados Ordinários - MQO e a regressão logística (HAIR et al., 2005). A regressão linear com o método MQO para a análise da presença de membros não familiares no Conselho de Administração, mensurada a partir da proporção de membros não familiares em relação ao número total de membros no Conselho de Administração da empresa. O modelo da regressão com o método MQO segue:

$$
\text { PCANF }=\beta_{0}+\beta_{1} \text { CONFLITO_PP }+\beta_{2} \text { CONFLITO_FB }+\beta_{3} \text { IE }+\beta_{4} \text { TAM }+\beta_{5} \mathrm{~N} 1+\beta_{6} \mathrm{~N} 2+\beta_{7} \mathrm{NM}+\beta_{8} \mathrm{AA}+\varepsilon
$$

Para a regressão linear com o método MQO, observaram-se alguns pressupostos como o da normalidade, da ausência de multicolinearidade e ausência de heterocedasticidade (FÁVERO et al., 2009).

A regressão logística foi utilizada para a análise da presença de membros não familiares no Conselho de Administração, mensurada a partir da variável dummy, codificando 1 se o presidente do Conselho de Administração da empresa era membro não familiar e 0 caso contrário. $O$ modelo da regressão logística segue:

$$
\text { PRCANF }=\beta_{0}+\beta_{1} \text { CONFLITO_PP }+\beta_{2} \text { CONFLITO_FB }+\beta_{3} \text { IE }+\beta_{4} \text { TAM }+\beta_{5} \mathrm{~N} 1+\beta_{6} \mathrm{~N} 2+\beta_{7} \mathrm{NM}+\beta_{8} \mathrm{AA}+\varepsilon
$$

Para a regressão logística, realizaram-se algumas medidas de ajustamento do modelo, como o Pseudo $\mathrm{R}^{2}$ e o Teste de Hosmer-Lemeshow (FÁVERO et al., 2009), apresentados ao longo da análise dos dados

\section{Análise dos Resultados}

\subsection{Análise descritiva}

A Tabela 2 apresenta a análise descritiva das variáveis, a partir da média, desvio padrão, mínimo e máximo. As empresas familiares da amostra em média foram fundadas há 50 anos, tem seus ativos totais avaliados em $\mathrm{R} \$ 5,9$ bilhões e faturam $\mathrm{R} \$ 4,9$ bilhões por ano. Os acionistas familiares detêm $67,19 \%$ das ações ON contra apenas 32,81\% para acionistas não familiares. Em algumas empresas, a família possui a totalidade das ações ON, tendo assim total controle e poder de voto nas assembleias da empresa.

No Conselho de Administração, a presença de membros não familiares é maior do que a presença de membros familiares, sendo $59 \%$ a proporção de membros não familiares e $41 \%$ de membros familiares. Em 26,20\% das empresas o Presidente do Conselho de Administração não é membro da família.

Em relação ao conflito principal-principal, o maior acionista possui em média $50,46 \%$ das ações ON e o segundo maior acionista possui em média $15,81 \%$ das ações ON. No que se refere ao conflito family blockholder, verificou-se que em $31,03 \%$ das empresas há mais de uma família controladora. Quanto ao nível de governança, $6,89 \%$ das empresas familiares estão no nível 1, 4,82\% no nível 2, 33,10\% no novo mercado e $44,82 \%$ possuem acordo de acionistas.

\begin{tabular}{|c|c|c|c|c|c|}
\hline & Média & Desvio padrão & $\begin{array}{c}\text { Coeficiente de } \\
\text { Variação }\end{array}$ & Min & Max \\
\hline \multicolumn{6}{|l|}{ Composição do Conselho de Administração } \\
\hline $\begin{array}{l}\text { PCANF } \\
\text { PRCANF a }\end{array}$ & 0,59 & 0,25 & 0,42 & 0 & 1 \\
\hline \multicolumn{5}{|l|}{ Conflitos de Agência } & - \\
\hline CONFLITO_PP & 1,72 & 1 & 0,58 & $-1,76$ & 2,00 \\
\hline CONFLITO_FB a & $0,31^{*}$ & - & - & - & - \\
\hline \multicolumn{6}{|l|}{ Variáveis de controle } \\
\hline IE & 50,85 & 32,61 & 0,64 & 10 & 149 \\
\hline TAM $^{\mathrm{b}}$ & 4.901 & 19.423 & 3,96 & 0,04 & 181.680 \\
\hline $\mathrm{N} 1^{\mathrm{a}}$ & $0,06^{*}$ & - & - & - & - \\
\hline $\mathrm{N} 2^{\mathrm{a}}$ & $0,04^{*}$ & - & - & - & - \\
\hline $\mathrm{NM}^{\mathrm{a}}$ & $0,33^{*}$ & - & - & - & - \\
\hline $\mathrm{AA}^{\mathrm{a}}$ & $0,44^{*}$ & - & - & - & - \\
\hline
\end{tabular}

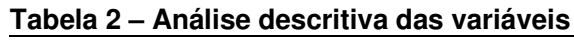

Nota. $\mathrm{N}=145 .{ }^{a}$ Variável dummy. ${ }^{\mathrm{b}} \mathrm{A}$ variável TAM foi apresentada na Tabela 2 em milhões de reais, no entanto no modelo de regressão foi utilizada com medida logarítmica, conforme Tabela 1. * A média das variáveis dummy foi utilizada para medir a proporção de elementos do conjunto que possuía aquela característica. PCANF: proporção de membros não familiares no Conselho de Administração; PRCANF: Presidente do Conselho de Administração não é da família; CONFLITO_PP: conflito principal-principal; CONFLITO_FB: conflito family blockholder, IE: idade empresa; TAM: tamanho; N1: nível 1 de governança; N2: nível 2 de governança; NM: novo mercado; AA: acordo de acionistas.

Fonte: Dados da pesquisa.

A Tabela 3 apresenta a matriz de correlação com as principais variáveis do estudo. Foram realizadas a correlação de Pearson e a correlação de Spearman. Referente a correlação de Pearson, nota-se, numa 
perspectiva bivariada, correlação significativa em até $1 \%$ entre o conflito de agência principal-principal (CONFLITO_PP) e a proporção de membros não familiares no Conselho de Administração (PCANF) (0,2146), denotando que quanto maior o conflito de agência principal-principal menor a proporção de membros não familiares no Conselho de Administração, o que vai ao encontro da hipótese 1 do estudo.

Ademais, constatou-se a correlação significativa em até $5 \%$ entre as variáveis tamanho (TAM) e $\operatorname{PCANF}(0,1810)$, sugerindo que quanto maior o tamanho da empresa em relação ao logaritmo da receita de vendas maior a proporção de membros não familiares no Conselho de Administração. Contataram-se, também, correlações significativas em até $10 \%$ entre idade da empresa (IE) e PCANF $(-0,1528)$, indicando que quanto mais antiga a empresa menor a proporção de membros não familiares no Conselho de Administração.

Para a correlação de Spearman, verificaram-se correlações significativas em até $5 \%$ entre PCANF e CONFLITO_PP $(-0,1914)$ e entre PCANF e IE $(-0,1916)$, o que corrobora com os resultados da correlação de Pearson para as mesmas variáveis.

Tabela 3 - Matriz de correlação

\begin{tabular}{|c|c|c|c|c|}
\hline & PCANF & CONFLITO PP & $\mathrm{IE}$ & TAM \\
\hline PCANF & 1 & $-0,1914^{\star \star}$ & $-0,1916^{\star *}$ & 0,1356 \\
\hline CONFLITO_PP & $-0,2146^{\star \star *}$ & 1 & 0,0309 & 0,0615 \\
\hline $\mathrm{IE}-$ & $-0,1528^{*}$ & 0,0262 & 1 & $-0,0090$ \\
\hline TAM & $0,1810^{* *}$ & 0,0186 & 0,0302 & 1 \\
\hline
\end{tabular}

Nota. $\mathrm{N}=145 .{ }^{*} \mathrm{p}<0.1 .{ }^{* *} \mathrm{p}<0.05 .{ }^{* * *} \mathrm{p}<0.001$. Triângulo superior corresponde a Correlação de Spearman e o triângulo inferior a Correlação de Pearson. PCANF: proporção de membros não familiares no Conselho de Administração; CONFLITO_PP: conflito principalprincipal; IE: idade empresa; TAM: tamanho.

Fonte: Dados da pesquisa.

\subsection{Resultados da Regressão Linear com base no MQO e Regressão Logística}

$\mathrm{Na}$ Tabela 4, apresentam-se os resultados da regressão linear com base no MQO, considerando como variável dependente a proporção de membros não familiares no Conselho de Administração (PCANF). Foram analisados dois modelos para a variável PCANF, o modelo 1 considerando apenas as variáveis de controle e o modelo 2 com a inserção das variáveis independentes. Referente ao $\mathrm{R}^{2}$, o modelo 1 apresentou $8,45 \%$ e o modelo 2 apresentou $11,94 \%$. Quanto ao $R^{2}$ ajustado, o modelo 1 apresentou $4,47 \%$ e o modelo 2 apresentou $6,76 \%$.

A partir dos resultados da Tabela 4, verifica-se que considerando o modelo 1 , a variável NM apresentou a relação significativa e positiva em até $10 \%$ com PCANF, indicando que em empresas presentes no nível de governança do Novo Mercado (NM), há maior proporção de membros não familiares no Conselho de Administração. Tal resultado confirma o que descreve o estudo de Karam, Machado Filho e Abib (2019) que, no contexto brasileiro, a presença de conselheiros independentes é requisito legal para empresas de capital aberto que estão listadas no segmento do NM, logo em empresas familiares entende-se que esses conselheiros independentes sejam membros não familiares.

Tabela 4 - Regressão linear com base no MQO

\begin{tabular}{|c|c|c|c|c|c|}
\hline & & \multicolumn{4}{|c|}{$\begin{array}{c}\text { PCANF } \\
\text { Equação (1) }\end{array}$} \\
\hline & & \multicolumn{2}{|c|}{ Modelo 1} & \multicolumn{2}{|c|}{ Modelo 2} \\
\hline & & Coef. & Std. Err. & Coef. & Std. Err. \\
\hline CONFLITO_PP & $\mathrm{H} 1(-)$ & & & $-0,0450^{\star \star}$ & 0,0224 \\
\hline CONFLITO_FB ${ }^{a}$ & $\mathrm{H} 2(+)$ & & & 0,0427 & 0,0446 \\
\hline IE & & $-0,0007$ & 0,0007 & $-0,0009$ & 0,0007 \\
\hline TAM & & 0,0100 & 0,0090 & 0,0126 & 0,0092 \\
\hline $\mathrm{N} 1^{\mathrm{a}}$ & & 0,0372 & 0,0895 & 0,0265 & 0,0886 \\
\hline $\mathrm{N} 2^{\mathrm{a}}$ & & $-0,0033$ & 0,1016 & 0,0010 & 0,1005 \\
\hline $\begin{array}{l}N^{a} \\
A^{a}\end{array}$ & & 0,0986* & 0,0542 & 0,0677 & 0,0560 \\
\hline $\begin{array}{l}\mathrm{AA}^{\mathrm{a}} \\
\text { const }\end{array}$ & & $0,3848^{\star *}$ & $\begin{array}{l}0,0432 \\
0,1690\end{array}$ & 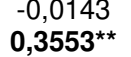 & $\begin{array}{l}0,0447 \\
0,1684\end{array}$ \\
\hline Prob > F & & \multicolumn{2}{|c|}{0,0545} & \multicolumn{2}{|c|}{0,0238} \\
\hline & & \multicolumn{2}{|c|}{0,0845} & \multicolumn{2}{|c|}{0,1194} \\
\hline $\mathbf{R}^{2}$ ajustado & & \multirow{2}{*}{\multicolumn{2}{|c|}{0,0447}} & \multicolumn{2}{|c|}{0,0676} \\
\hline VIF & & & & \multicolumn{2}{|c|}{1,71} \\
\hline
\end{tabular}

Nota. $\mathrm{N}=145 .{ }^{*} \mathrm{p}<0.1$. ${ }^{* *} \mathrm{p}<0.05$. ${ }^{a}$ Variável dummy. PCANF: proporção de membros não familiares no Conselho de Administração; CONFLITO_PP: conflito principal-principal; CONFLITO_FB: conflito family blockholder, IE: idade empresa; TAM: tamanho; N1: nível 1 de governança; N2: nível 2 de governança; NM: novo mercado; AA: acordo de acionistas. Realizou-se o teste do pressuposto da normalidade dos resíduos a partir do teste Shapiro Francia, que indicou uma significância de 0,0125 para o Modelo 1 e 0,0327 para o Modelo 2. Conforme o número de observações e considerando o Teorema Central do Limite, relaxa-se o pressuposto da normalidade, conforme Freund e Simon (2000). Quanto ao pressuposto de homocedasticidade, utilizou-se o teste de White, que apresentou ausência de heterocedasticidade, com significância igual à 0,0779 para o Modelo 1 e 0,5470 para o Modelo 2 .

Fonte: Dados da pesquisa. 
Quanto ao modelo 2, constata-se que, para a proxy PCANF apenas CONFLITO_PP, se demonstrou significativa para um nível de significância de $5 \%$, apontando que quanto maior o conflito principal-principal menor a proporção de membros não familiares no Conselho de Administração, o que confirma a relação negativa indicada na matriz de correlação na Tabela 3.

Esse resultado corrobora com o estudo de Kabbach de Castro, Aguilera e Crespí-Cladera (2017) que, quando a família possui mais participações na empresa, os membros da família possuem a expectativa de controlar os processos de tomada de decisão de modo a preservar sua influência, o que faz com que os proprietários da família construam mecanismos de governança que fortaleçam seu poder, o que não ocorre com o ingresso de membros não familiares no Conselho de Administração.

Com o intuito de aumentar a robustez dos dados, aplicou-se tratamento adicional no modelo 2 da Eq. (1) que analisa a proporção de membros não familiares no Conselho de Administração (PCANF). Para tanto, empregou-se a regressão TOBIT em substituição da regressão linear com base no MQO para verificar se o intervalo restrito da variável dependente proporcionava inconsistências nos parâmetros do modelo; no entanto, não se constataram diferenças significativas nos resultados da regressão TOBIT em comparação com a regressão linear baseada no MQO. Para a proxy CONFLITO_PP, o coeficiente foi de -0,0463, com significância até $5 \%$, enquanto a proxy CONFLITO_FB apresentou coeficiente de 0,0473 , não sendo significativa.

A Tabela 5 apresenta os resultados da regressão logística, considerando como variável dependente a variável indicativa de que o presidente do Conselho de Administração não é da família (PRCANF). Analisaram-se dois modelos: o modelo 1 considerando apenas as variáveis de controle e o modelo 2 com a inserção das variáveis independentes. Quanto ao Pseudo R², o modelo 1 apresentou $4,41 \%$ e o modelo 2 apresentou $9,45 \%$.

A partir da análise da Tabela 5, nota-se que para o modelo 1 nenhuma variável se apresentou significativa com PRCANF. Enquanto para o modelo 2, verifica-se que CONFLITO_PP e CONFLITO_FB apresentaram relação significativa com PRCANF, de forma negativa, em até 5\%, e positiva, em até $10 \%$, respectivamente.

Dessa forma, entende-se que empresas que possuem maior conflito de agência entre principalprincipal apresentam menor a probabilidade de o Presidente do Conselho de Administração não ser da família e quanto maior o conflito family blockholder, maior a probabilidade de o Presidente do Conselho de Administração não ser da família.

Tabela 5 - Regressão Logística

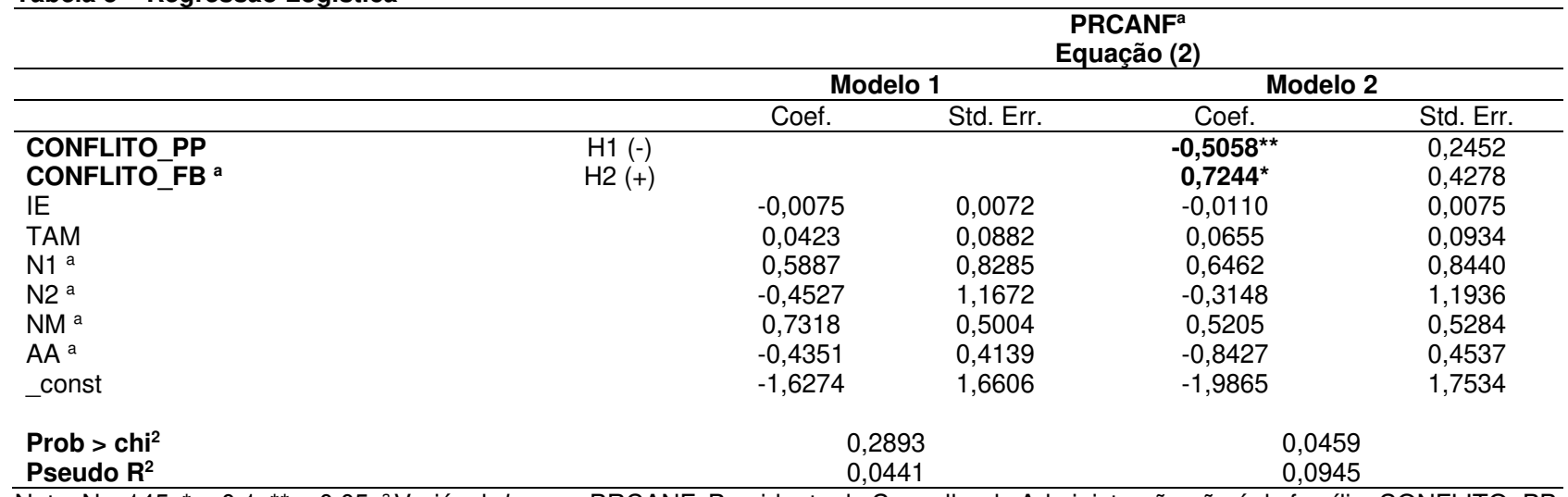

Nota. $\mathrm{N}=145 .{ }^{*} \mathrm{p}<0.1 .{ }^{* *} \mathrm{p}<0.05 .{ }^{a}$ Variável dummy. PRCANF: Presidente do Conselho de Administração não é da família; CONFLITO_PP: conflito principal-principal; CONFLITO_FB: conflito family blockholder, IE: idade empresa; TAM: tamanho; N1: nível 1 de governança; N2: nível 2 de governança; NM: novo mercado; AA: acordo de acionistas. O Teste de Hosmer-Lemeshow apresentou significância de 0,3994 para o Modelo 1 e de 0,2660 para o Modelo 2, indicando que não há diferenças significativas entre as frequências previstas e as observadas, ao nível de significância de $5 \%$.

Fonte: Dados da pesquisa.

A partir dos resultados da Regressão Linear com base no MQO e da Regressão Linear Logística, parte-se para a aceitação das hipóteses $\mathrm{H} 1$ e H2, conforme apresenta a Tabela 6 . A hipótese H1, que trata sobre uma relação negativa entre o conflito principal-principal e a presença de membros não familiares no Conselho de Administração, foi aceita considerando o nível de significância de $5 \%$. Nesse sentido, tanto a proporção de membros não familiares quanto a Presidência do Conselho de Administração são negativamente significantes.

Assim, os resultados corroboram com a perspectiva de que nas empresas familiares com alta concentração acionária, ou seja, em que há conflitos de agência tipo principal-principal, há indicativos de menor participação de membros não familiares no Conselho de Administração, conforme traz o estudo de Vazquez, Carrera e Cornejo (2020). Nesse sentido, os resultados indicam que nas empresas familiares há a expectativa por parte dos membros familiares de manter seu controle e influência na tomada de decisões (KABBACH DE CASTRO; AGUILERA; CRESPÍ-CLADERA, 2017), o que limita a presença de membros não 
familiares nos Conselhos de Administração, pois consequentemente isso reduziria o controle da família nos negócios, à medida que os membros não familiares garantem uma maior proteção dos interesses dos acionistas minoritários no Conselho de Administração (MORCK; YEUNG, 2003; PENG, 2004; RHOADES; RECHNER, 2001).

Tabela 6 - Resumo das hipóteses

\begin{tabular}{c|l|c|c}
\hline \multicolumn{1}{c|}{ Tabela 6 - Rescrição } & \multicolumn{2}{c}{ Resultado } \\
\cline { 3 - 4 } Hipótese & \multicolumn{1}{c}{ PCANF } & PRCANF \\
\hline H1 & $\begin{array}{l}\text { Há uma associação negativa entre o conflito principal-principal e a presença de membros } \\
\text { não familiares no Conselho de Administração de empresas familiares de capital aberto. }\end{array}$ & Suportada & Suportada \\
\hline H2 & $\begin{array}{l}\text { Há uma associação positiva entre o conflito family blockholder e a presença de } \\
\text { membros não familiares no Conselho de Administração de empresas familiares de } \\
\text { capital aberto. }\end{array}$ & $\begin{array}{c}\text { Não } \\
\text { suportada }\end{array}$ & Suportada \\
\hline
\end{tabular}

Fonte: Dados da pesquisa.

A hipótese $\mathrm{H} 2$, que trata sobre uma relação positiva entre o conflito family blockholder e a presença de membros não familiares no Conselho de Administração, foi aceita considerando o nível de significância de $10 \%$. No entanto, apenas a proxy relativa à Presidência do Conselho de Administração se apresentou significativa.

Assim, verifica-se que em empresas multifamiliares quando há conflitos entre as diferentes famílias proprietárias, opta-se pela presença de membros não familiares no Conselho de Administração, com o intuito de facilitar o alinhamento e a negociação dos diferentes objetivos entre as famílias proprietárias e evitar a interferência descoordenada dessas no negócio (ZELLWEGER; KAMMERLANDER, 2015; HIEBL; LI, 2018).

\section{Conclusões}

Este estudo objetivou analisar a influência dos conflitos de agência decorrentes da estrutura acionária acerca da presença de membros não familiares no Conselho de Administração das empresas familiares brasileiras de capital aberto.

A partir dos resultados, verificou-se que há uma relação negativa e significativa no nível de $5 \%$ entre o conflito principal-principal e a presença de membros não familiares no Conselho de Administração de empresas familiares. Nesse sentido, o estudo apresenta que a alta concentração de propriedade por uma família que provoca o conflito de agência principal-principal, leva a uma menor presença de membros não familiares no Conselho de Administração, para não ocasionar o enfraquecimento do controle da família na empresa.

Ademais, verificou-se que em empresas multifamiliares, em que há presença de mais de uma família controladora se apresentou positiva e significativa no nível de $10 \%$, indicando que o conflito family blockholder influencia de forma significativa a presença de membros não familiares no Conselho de Administração. Diante disso, o estudo ressalta que a presença de membros não familiares no Conselho de Administração pode ser considerado um mecanismo de governança que auxilia a redução do conflito de agência family blockholder nas empresas familiares brasileiras de capital aberto. O Conselho de Administração ser presidido por um membro não familiar permite um melhor alinhamento dos interesses entre as diferentes famílias que controlam a empresa.

Este estudo contribui para a literatura, uma vez que trabalha com a relação entre os conflitos de agência decorrentes da estrutura acionária e a composição do Conselho de Administração nas empresas familiares, sendo que poucas pesquisas analisaram como os problemas de agência estão associados à presença de executivos não-familiares. Além do mais, o estudo contribui para a literatura sobre empresas familiares e problemas de agência (governança corporativa), tendo em vista que se trata de problemas de agência específicos das empresas familiares, permitindo uma melhor compreensão dos tipos de conflitos principal-principal e family blockholders os quais ocorrem nas empresas familiares de capital aberto no Brasil. Nesse sentido, o estudo contribui com a literatura ao investigar como as especificidades e as particularidades das empresas familiares afetam a maneira que são gerenciadas e organizadas, conforme Gomez-Mejia et al. (2020).

Ademais, este artigo aborda o conflito principal-principal no contexto de Brasil, caracterizado pela alta concentração da estrutura de propriedade nas empresas familiares, como a maior parte dos países emergentes da América Latina (BRIANO-TURRENT; POLETTI-HUGHES, 2017). O estudo de mecanismos de governança nesse contexto é importante para trazer novos insights das empresas familiares em mercados emergentes, em que não há um forte sistema institucional para proteção dos acionistas minoritários. No estudo de Vazquez, Carrera e Cornejo (2020) há o incentivo de investigações sobre essa temática numa ampla gama de contextos.

Quanto ao conflito family blockholder, o estudo apresenta relevância ao tratar de um tipo de conflito que é específico de empresas multifamiliares, que são empresas ainda pouco estudadas. Ao investigar como se comportam esse tipo específico de empresa em relação aos conflitos de agência, o estudo contribui com 
a literatura na compreensão da heterogeneidade das empresas familiares, conforme argumenta Duran e Ortiz (2020).

Como limitações, destacam-se as proxies utilizadas para medir os conflitos, que podem não captar totalmente o fenômeno. Para pesquisas futuras, recomenda-se o estudo de outras variáveis/proxies para se verificar outros fatores que são determinantes para a presença de membros não familiares no Conselho de Administração das empresas familiares brasileiras e outros tipos de proxies podem ser utilizadas para medir as possibilidades de conflitos. Além disso, uma análise qualitativa dos dados também permitiria um outro olhar para as relações e o contexto, trazendo um aprofundamento que não é possível na pesquisa quantitativa.

\section{Referências}

BENNEDSEN, M.; NIELSEN, K. M.; PEREZ-GONZLEZ, F.; WOLFENZON, D. Inside the Family Firm: The Role of Families in Succession Decisions and Performance. Quarterly Journal of Economics, v. 122, n. 2, p. 647-691, 2007. DOI: https://doi.org/10.1162/qjec.122.2.647

BERENBEIM, R. E. How Business Families Manage the Transition from Owner to Professional Management. Family Business Review, v. 3, n. 1, p. 69-110, 1990. DOI: https://doi.org/10.1111/i.1741$\underline{6248.1990 .00069 . x}$

BERTRAND, M.; JOHNSON, S.; SAMPHANTHARAK, K.; SCHOAR, A. Mixing family with business: A study of Thai business groups and the families behind them. Journal of Financial Economics, v. 88, p. 466-498, 2008. DOI: https://doi.org/10.1016/.j.fineco.2008.04.002

BLUMENTRITT, T. P.; KEYT, A. D.; ASTRACHAN, J. H. Creating an Environment for Successful Nonfamily CEOs: An Exploratory Study of Good Principals. Family Business Review, v. 20, n. 4, p. 321-335, 2007. DOI: https://doi.org/10.1111/j.1741-6248.2007.00102.x

BRASIL BOLSA BALCÃO (B3). Comparativo dos segmentos de listagem. Disponível em: http://www.b3.com.br/pt br/produtos-e-servicos/solucoes-para-emissores/segmentos-de-listagem/sobresegmentos-de-listagem/. Acesso em: 19 jun. 2020.

BRESSAN, A. A.; SCHIEHLL, E.; PROCIANOY, J. L.; CASTRO, L. R. K. Perspectivas da Pesquisa em Governança de Empresas Familiares no Brasil. Revista de Administração Contemporânea, v. 23, n. 6, p. 696-702, 2019. DOI: https://doi.org/10.1590/1982-7849rac2019190331

BRIANO-TURRENT, G. C.; POLETTI-HUGHES, J. Corporate governance compliance of family and nonfamily listed firms in emerging markets: Evidence from Latin America. Journal of Family Business Strategy, v. 8, n. 4, p. 237-247, 2017. DOI: https://doi.org/10.1016/j.jfbs.2017.10.001

CARNEY, M. Corporate governance and competitive advantage in family-controlled firms.

Entrepreneurship Theory and Practice, v. 29, n. 3, p. 246-265, 2005. DOI: https://doi.org/10.1111/j.1540$\underline{6520.2005 .00081 . x}$

CHRISMAN, J. J.; CHUA, J. H.; LITZ, R. A. Comparing the agency costs of family and non-family firms: Conceptual issues and exploratory evidence. Entrepreneurship Theory and Practice, v. 28, n. 4, p. 335354, 2004. DOI: https://doi.org/10.1111/j.1540-6520.2004.00049.x

CHUA, J. H.; CHRISMAN, J. J.; SHARMA, P. Defining the family business by behavior. Entrepreneurship Theory and Practice, v. 23, n. 4, p. 19-39, 1999. DOI: https://doi.org/10.1177/104225879902300402

COSTA, L.; SAMPAIO, J.; FLORES, E. S. Diversidade de gênero nos conselhos desempenho e risco financeiro nas empresas familiares. Revista de Administração Contemporânea, v. 23, n. 6, p. 721-738, 2019. DOI: https://doi.org/10.1590/1982-7849rac2019180327

DURAN, P.; ORTIZ, M. When More Is Better: Multifamily Firms and Firm Performance. Entrepreneurship Theory and Practice, v. 44, n. 4, p. 761-783, 2020. DOI: https://doi.org/10.1177/1042258719851206

EISENHARDT, K. Agency theory: An assessment and review. Academy of Management Review, v. 14, n. 1, p. 57-74, 1989. DOI: https://doi.org/10.2307/258191

FANG, H. C.; RANDOLPH, R. V.; MEMILI, E.; CHRISMAN, J. J. Does size matter? The moderating effects of firm size on the employment of nonfamily managers in privately held family SMEs. Entrepreneurship Theory and Practice, v. 40, n. 5, p. 1017-1039, 2016. DOI: https://doi.org/10.1111/etap.12156 
FÁVERO, L. P.; BELFIORE, P.; SILVA, F. L.; CHAN, B. L. Análise de dados: modelagem multivariada para tomar decisões. Rio de Janeiro, Elsevier, 2009.

FREUND, J. E.; SIMON, G. A. Estatística aplicada: Economia, Administração e Contabilidade. Porto Alegre, Bookman, 2000.

GARCIA-CASTRO, R.; CASASOLA, M. J. A set-theoretic analysis of the components of family involvement in publicly listed and major unlisted firms. Journal of Family Business Strategy, v. 2, n. 1, p. 15-25, 2011. DOI: https://doi.org/10.1016/i.jfbs.2011.01.002

GOMEZ-MEJIA, L.; BASCO, R.; GONZALEZ, A. C.; MULLER, C. G. Family business and local development in Iberoamerica. Cross Cultural \& Strategic Management, 2020. DOI: https://doi.org/10.1108/CCSM-02$\underline{2020-223}$

HAIR, J. F.; TATHAM, R. L.; ANDERSON, R. E.; BLACK, W. Análise multivariada de dados. 5. ed. Porto Alegre: Bookman, 2005.

HIEBL, M. R. W. A finance professional who understands the family: Family firms' specific requirements for non-family chief financial officers. Review of Managerial Science, v. 8, n. 4, p. 465-494, 2014. DOI: https://doi.org/10.1007/s11846-013-0112-6

HIEBL, M. R.; LI, Z. Non-Family managers in family firms: review, integrative framework and future research agenda. Review of Managerial Science, p. 1-45, 2018. DOI: https://doi.org/10.1007/s11846-018-0308-x

HILLIER, D.; MCCOLGAN, P. Firm Performance and Managerial Succession in Family Managed Firms. Journal of Business Finance \& Accounting, v. 36, n. 3-4, p. 461-484, 2009. DOI: https://doi.org/10.1111/j.1468-5957.2009.02138.x

ILHAN-NAS, T.; OKAN, T.; TATOGLU, E.; DEMIRBAG, M.; WOOD, G.; GLAISTER, K. W. Board composition, family ownership, institutional distance and the foreign equity ownership strategies of Turkish MNEs. Journal of World Business, v. 53, n. 6, p. 862-879, 2018. DOI:

https://doi.org/10.1016/j.jwb.2018.07.006

INSTITUTO BRASILEIRO DE GOVERNANÇA CORPORATIVA (IBGC). Governança em empresas familiares: evidências brasileiras. São Paulo, SP, Brasil, 2019.

JENSEN, M. C.; MECKLING, W. H. Theory of the firm: Managerial behavior, agency costs and ownership structure. Journal of Financial Economics, v. 3, n. 4, p. 305-360, 1976. DOI: https://doi.org/10.1016/0304$\underline{405 X(76) 90026-X}$

KABBACH DE CASTRO, L. R.; AGUILERA, R. V.; CRESPÍ-CLADERA, R. Family firms and compliance: Reconciling the conflicting predictions within the socioemotional wealth perspective. Family Business Review, v. 30, n. 2, p. 137-159, 2017. DOI: https://doi.org/10.1177/0894486516685239

KARAM, P. B. S.; MACHADO FILHO, C. A. P.; ABIB, G. Conflicts in boards of family firms: A theoretical framework for strategic decision-making. Revista de Administração Contemporânea, v. 23, n. 6, p. 703720, 2019. DOI: https://doi.org/10.1590/1982-7849rac2019190083

KLEINERT, V. C.; LANA, J.; FLORIANI, D. Governança Corporativa llimitada para Empresas Limitadas. Revista de Administração Contemporânea, v. 23, n. 6, p. 807-811, 2019. DOI: https://doi.org/10.1590/1982-7849rac2019180082

LAFFRANCHINI, G.; BRAUN, M. Slack in family firms: evidence from Italy (2006-2010). Journal of Family Business Management, v. 4, n. 2, p. 171-193, 2014. DOI: https://doi.org/10.1108/JFBM-04-2013-0011

LIN, S.; HU, S. A Family Member or Professional Management? The Choice of a CEO and Its Impact on Performance. Corporate Governance: An International Review, v. 15, n. 6, p. 1348-1362, 2007. DOI: https://doi.org/10.1111/j.1467-8683.2007.00650.x

MENDONÇA, F. F. P.; MARTINS, H. C.; TERRA, P. R. S. Estrutura de Capital e Mecanismos de Governança Externos à Firma: Uma Análise Multipaís. Revista de Administração Contemporânea, v. 23, n. 6, p. 765-785, 2019. DOI: https://doi.org/10.1590/1982-7849rac2019190109 
MORCK, R.; YEUNG, B. Agency problems in large family business groups. Entrepreneurship Theory and Practice, v. 27, n. 4, p. 367-382, 2003. DOI: https://doi.org/10.1111/1540-8520.t01-1-00015

PENG, M. W. Outside directors and firm performance during institutional transitions. Strategic Management Journal, v. 25, p. 453-47, 2004. DOI: https://doi.org/10.1002/smi.390

PIEPER, T. M.; SMITH, A. D.; KUDLATS, J. ASTRACHAN, J. H. Article Commentary: The Persistence of Multifamily Firms: Founder Imprinting, Simple Rules, and Monitoring Processes. Entrepreneurship Theory and Practice, v. 39, n. 6, p. 1313-1337, 2015. DOI: https://doi.org/10.1111/etap.12179

PURKAYASTHA, S.; VELIYATH, R.; GEORGE, R. The roles of family ownership and family management in the governance of agency conflicts. Journal of Business Research, v. 98, n. 1, p. 50-64, 2019. DOI: https://doi.org/10.1016/i.jbusres.2019.01.024

RENDERS, A.; GAEREMYNCK, A. Corporate governance, principal-principal agency conflicts and firm value in European listed companies. Corporate Governance: An International Review, v. 20, n. 2, p. 125-143, 2012. DOI: https://doi.org/10.1111/j.1467-8683.2011.00900.x

RHOADES, D. L.; RECHNER, P. L. The role of ownership and corporate governance factors in international entry mode selection. The International Journal of Organizational Analysis, v. 9, n. 4, p. 309-326, 2001. DOI: https://doi.org/10.1108/eb028938

SCHULZE, W. S.; LUBATKIN, M. H.; DINO, R. N. Toward a theory of agency and altruism in family firms. Journal of Business Venturing, v. 18, n. 1, p. 473-490, 2003. DOI: https://doi.org/10.1016/S0883$\underline{\text { 9026(03)00054-5 }}$

SELEKLER-GOKSEN, N. N.; YILDIRIM-ÖKTEM, Ö. Countervailing institutional forces: Corporate governance in Turkish family business groups. Journal of Management Governance, v. 13, p. 193-213, 2009. DOI: https://doi.org/10.1007/s10997-009-9083-z

SINGLA, C.; VELIYATH, R.; GEORGE, R. Family firms and internationalization-governance relationships: Evidence of secondary agency issues. Strategic Management Journal, v. 35, n. 4, p. 606-616, 2014. DOI: https://doi.org/10.1002/smj.2111

SHYU, J. Family ownership and firm performance: evidence from Taiwanese firms. International Journal of Managerial Finance, v. 7, n. 4, p. 397-411, 2011. DOI: https://doi.org/10.1108/174391311111166393

TABOR, W.; CHRISMAN, J. J.; MADISON, K.; VARDAMAN, J. M. Nonfamily Members in Family Firms: A Review and Future Research Agenda. Family Business Review, v. 31, n. 1, p. 54-79, 2018. DOI: https://doi.org/10.1177/0894486517734683

VAZQUEZ, P.; CARRERA, A.; CORNEJO, M. Corporate governance in the largest family firms in Latin America. Cross Cultural \& Strategic Management, 2020. DOI: https://doi.org/10.1108/CCSM-11-2018$\underline{0194}$

VILLALONGA, B.; AMIT, R. How do family ownership, control and management affect firm value? Journal of Financial Economics, v. 80, n. 2, p. 385-417, 2006. DOI: https://doi.org/10.1016/j.jineco.2004.12.005

YOUNG, M. N.; PENG, M. W.; AHLSTROM, D.; BRUTON, G. D.; JIANG, Y. Corporate governance in emerging economies: A review of the principal-principal perspective. Journal of Management Studies, v. 45, n. 1, p. 196-220, 2008. DOI: https://doi.org/10.1111/i.1467-6486.2007.00752.x

ZELLWEGER, T.; KAMMERLANDER, N. Family, wealth, and governance: an agency account. Entrepreneurship Theory and Practice, v. 39, n. 6, p. 1281-1303, 2015. DOI: http://dx.doi.org/10.1111/etap.12182

ZONA, F. CEO leadership and board decision processes in family-controlled firms: comparing family and non-family CEOs. Small Business Economics, v. 47, n. 3, p. 735-753, 2016. DOI:

https://doi.org/10.1007/s11187-016-9764-3 


\section{NOTAS}

\section{AGRADECIMENTOS}

Agradecimentos à Coordenação de Aperfeiçoamento de Pessoal de Nível Superior (CAPES) e ao Professor Dr. Moacir Manoel Rodrigues Junior.

\section{CONTRIBUIÇÃO DE AUTORIA}

Concepção e elaboração do manuscrito: C. Adam, J. K. Tene, D. M. Mucci, F. Beck

Coleta de dados: C. Adam, J. K. Tene

Análise de dados: C. Adam, J. K. Tene, D. M. Mucci, F. Beck

Discussão dos resultados: C. Adam, J. K. Tene, D. M. Mucci, F. Beck

Revisão e aprovação: D. M. Mucci, F. Beck

\section{CONJUNTO DE DADOS DE PESQUISA}

O conjunto de dados que dá suporte aos resultados deste estudo não está disponível publicamente.

\section{FINANCIAMENTO}

O presente trabalho foi realizado com apoio da Coordenação de Aperfeiçoamento de Pessoal de Nível Superior - Brasil (CAPES) - Código de Financiamento 001.

\section{CONSENTIMENTO DE USO DE IMAGEM}

Não se aplica.

\section{APROVAÇÃO DE COMITÊ DE ÉTICA EM PESQUISA}

Não se aplica.

\section{CONFLITO DE INTERESSES}

Não se aplica.

\section{LICENÇA DE USO}

Os Direitos Autorais para artigos publicados neste periódico são do autor, com direitos de primeira publicação para a Revista. Em virtude de aparecerem nesta Revista de acesso público, os artigos são de uso gratuito, com atribuições próprias, em aplicações educacionais, de exercício profissional e para gestão pública. A Revista adotou a licença Creative Commons Atribuição 4.0 Internacional - CC BY NC ND. Esta licença permite acessar, baixar (download), copiar, imprimir, compartilhar, reutilizar e distribuir os artigos desde que com a citação da fonte, atribuindo os devidos créditos de autoria. Nesses casos, nenhuma permissão é necessária por parte dos autores ou dos editores. Autores têm autorização para assumir contratos adicionais separadamente, para distribuição não-exclusiva da versão do trabalho publicada nesta revista (ex.: publicar em repositório institucional ou um capítulo de livro).

\section{PUBLISHER}

Universidade Federal de Santa Catarina. Curso de Ciências Contábeis e Programa de Pós-graduação em Contabilidade. Publicação no Portal de Periódicos UFSC. As ideias expressadas neste artigo são de responsabilidade de seus autores, não representando, necessariamente, a opinião dos editores ou da universidade.

\section{EDITORES}

Carlos Eduardo Facin Lavarda e Suliani Rover

\section{HISTÓRICO}

Recebido em: 05/12/2019 - Revisado por pares em: 25/05/2020 - Reformulado em: 29/06/2020 Recomendado para publicação em: 31/07/2020 - Publicado em: 30/09/2020 